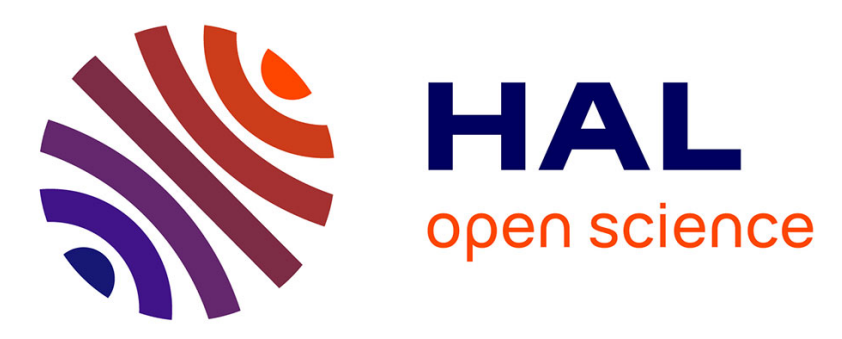

\title{
Design and Analysis of a Practical Codebook for Non-Coherent Communications
}

Ngo Khac-Hoang, Alexis Decurninge, Maxime Guillaud, Sheng Yang

\section{To cite this version:}

Ngo Khac-Hoang, Alexis Decurninge, Maxime Guillaud, Sheng Yang. Design and Analysis of a Practical Codebook for Non-Coherent Communications. 51st Asilomar Conference on Signals, Systems and Computers, 2017, Oct 2017, Pacific Grove, United States. pp.1237-1241, 10.1109/acssc.2017.8335549 . hal-01567048

\section{HAL Id: hal-01567048 \\ https://hal-centralesupelec.archives-ouvertes.fr/hal-01567048}

Submitted on 10 Jun 2020

HAL is a multi-disciplinary open access archive for the deposit and dissemination of scientific research documents, whether they are published or not. The documents may come from teaching and research institutions in France or abroad, or from public or private research centers.
L'archive ouverte pluridisciplinaire HAL, est destinée au dépôt et à la diffusion de documents scientifiques de niveau recherche, publiés ou non, émanant des établissements d'enseignement et de recherche français ou étrangers, des laboratoires publics ou privés. 


\title{
Design and Analysis of a Practical Codebook for Non-Coherent Communications
}

\author{
Khac-Hoang Ngo*†, Alexis Decurninge*, Maxime Guillaud*, Sheng Yang ${ }^{\dagger}$ \\ * Mathematical and Algorithmic Sciences Lab, Paris Research Center, Huawei Technologies, \\ 92100 Boulogne-Billancourt, France \\ Email: \{ngo.khac.hoang, alexis.decurninge, maxime.guillaud\} @huawei.com \\ ${ }^{\dagger}$ LSS, CentraleSupélec, 3 rue Joliot-Curie, 91190 Gif-sur-Yvette, France \\ Email: sheng.yang@centralesupelec.fr
}

\begin{abstract}
In this paper, we introduce and analyze a practical codebook for single-antenna non-coherent communications, with codewords belonging to the Grassmannian of lines defined on the complex vector space. This codebook is structured and has zero storage requirement. It has higher packing efficiency, represented by the minimum distance, than other structured codebooks in the literature. Numerical results show that this codebook outperforms the training-based scheme in terms of error probability and achievable rate.
\end{abstract}

\section{INTRODUCTION}

In wireless communication over fading channels, the instantaneous knowledge of channel state information (CSI) enables to adapt the transmission and reception to current channel conditions. The communication with a priori CSI at the receiver is said to be coherent. In practice, however, the channel coefficients are not granted for free prior to communication and need to be estimated at a cost that should not be ignored. Thus, communication without a priori CSI, also known as noncoherent communication [1], is a more reasonable framework.

We consider non-coherent communication over block fading channels such that the channel coefficients change independently between coherence blocks of $T \geq 2$ symbols. No $a$ priori CSI is available at either end of the channel. Specifically, we are interested in the single-input single-output (SISO) case, i.e., both the transmitter and the receiver have a single antenna. The non-coherent capacity, in bits per channel use, of this channel with Rayleigh fading was calculated for the high signal-to-noise ratio (SNR) regime in [2], [1] as

$$
C(\rho, T)=\frac{T-1}{T} \log _{2} \frac{\rho T}{e^{\gamma+1}}+\frac{1}{T} \log _{2} \frac{1}{(T-1) !}+o(1),
$$

when the SNR $\rho$ goes to infinity, where $\gamma \approx 0.5772$ is Euler's constant. The pre-log factor $\frac{T-1}{T}$ suggests that the non-coherent systems can still achieve a significant fraction of the coherent ergodic capacity $C_{\mathrm{coh}}(\rho)=\log _{2}\left(\rho / e^{\gamma}\right)+o(1)$, especially when $T$ is large. A natural scheme achieving this pre-log is based on channel training, in which one channel use is dedicated to transmit a reference symbol known to the receiver. The receiver estimates the channel from the noisy observation of this reference symbol, and then performs coherent detection on the remaining $T-1$ data symbols [3]. The performance of this approach, however, is always at a constant gap below the full capacity $C(\rho, T)[1]$.
It was shown in [2], [1] that the optimal strategy achieving the capacity $C(\rho, T)$ is to transmit an isotropically distributed vector on $\mathbb{C}^{T}$ during each coherence block and use the direction of this vector to carry information. The intuition behind this is that the channel coefficient $h$ only scales the transmitted signal vector without changing its direction. Therefore, upon normalization, the transmitted vector $x$ and the noise-free observation $h \boldsymbol{x}$ are identical in the Grassmannian of lines on $\mathbb{C}^{T}$. A number of Grassmannian constellation designs have been proposed (mostly for the multiple-antenna case at large) with different criteria, codebook generation and decoding methods. The codebooks generated by numerical optimization of max-min distance between codewords [4], [5] often exhibit good distance spectrum; however, the lack of structure imposes to store the whole codebook at both the transmitter and receiver, as well as to use a maximumlikelihood (ML) decoder at the receiver, which limits practical use to small codebooks. Other codebooks are generated with particular structures. For example, the Fourier codebook [6] contains the rows of the discrete Fourier transform (DFT) matrix with optimized frequencies. The exponential-mapped codebook [7] is obtained by mapping each coherent codeword $\boldsymbol{q} \in \mathbb{C}^{T-1}$ containing $T-1$ QAM symbols to a non-coherent codeword $\boldsymbol{x}=\left[\begin{array}{ll}\cos (\|\boldsymbol{q}\|) & -\frac{\sin (\|\boldsymbol{q}\|)}{\|\boldsymbol{q}\|} \boldsymbol{q}^{\top}\end{array}\right]^{\top}$.

In this work, we introduce a structured Grassmannian codebook for single-antenna non-coherent communications. Motivated by the relation between Grassmannian constellations and quantization codebooks for a Grassmannian source, we use the codebook generated by the cube-split quantizer proposed in [8] as a non-coherent constellation. We integrate soft demapping (using log-likelihood ratio) and compare the performance of this codebook with the training-based approach and some existing codebooks in the literature. We also derive the achievable rate of the system using this codebook.

The remainder of this paper is organized as follows. The system model is introduced in Section II. We describe the socalled cube-split codebook and maximum likelihood decoder in Section III, then calculate the bit-wise log-likelihood ratio and achievable rate associated with this codebook in Section IV. Numerical results on the error rates are provided in Section V. Finally, Section VI concludes this paper. 
In this paper, the Grassmannian manifold $G\left(\mathbb{C}^{T}, 1\right)$ is defined as the space of one-dimensional subspaces (lines) in $\mathbb{C}^{T}$. We use a vector $\boldsymbol{x} \in \mathbb{C}^{T}$ of unit Euclidean norm $(\|x\|=1)$ to represents the set $\{\lambda \boldsymbol{x}, \lambda \in \mathbb{C}\}$, which is a point in $G\left(\mathbb{C}^{T}, 1\right)$. We define the chordal distance between two Grassmannian lines represented by $x_{1}$ and $x_{2}$ as $d\left(x_{1}, x_{2}\right)=\sqrt{1-\left|x_{1}^{\mathrm{H}} x_{2}\right|^{2}}$.

\section{SYSTEM MODEL}

We consider a SISO non-coherent channel in which both the transmitter and receiver have a single antenna. The channel propagation is characterized by a scalar coefficient $h$ with arbitrary distribution ${ }^{1}$. The channel is block fading such that $h$ remains unchanged during each coherence block of $T>1$ symbols, and changes independently between blocks. Within a coherence block, the transmitter transmits a codeword $x \in \mathbb{C}^{T}$ satisfying $\mathbb{E}\left[\|x\|^{2}\right]=1$ at power $\rho$. The received signal is

$$
\boldsymbol{y}=\sqrt{\rho T} h \boldsymbol{x}+\boldsymbol{w},
$$

where $\boldsymbol{w} \sim \mathcal{C N}\left(0, \mathbf{I}_{T}\right)$ is the additive white Gaussian noise independent of $h$. We omit the block index for simplicity. Since the noise power is normalized, the transmit power $\rho$ is identified with the SNR at the receiver. The codeword $x$ is taken from a codebook $\mathcal{C}$ of finite cardinality $|\mathcal{C}|$. Let $N=\log _{2}(|\mathcal{C}|)$ be the number of bits per codeword.

\section{A. A baseline codebook with channel training}

Let us first consider a baseline codebook based on channel training. Each codeword $x$ begins with a reference symbol $x_{1}=\sqrt{\frac{\rho_{\tau}}{\rho T}}$ where $\rho_{\tau}$ is the training power, followed by $T-1$ data symbols $x_{i}=\sqrt{\frac{\rho T-\rho_{\tau}}{\rho T}} q_{i-1}, i=2, \ldots, T$ where $q_{i}$ are normalized quadrature amplitude modulation (QAM) symbols such that $\mathbb{E}\left[q_{i}\right]=1, i=1, \ldots, T-1$. Let $\boldsymbol{q}:=\left[q_{1}, \ldots, q_{T-1}\right]$. The receiver has the observation

$$
\boldsymbol{y}=\left[\begin{array}{ll}
\sqrt{\rho_{\tau}} h & \sqrt{\rho T-\rho_{\tau}} h \boldsymbol{q}^{\top}
\end{array}\right]^{\top}+\boldsymbol{w},
$$

and performs a scalar minimum mean square error (MMSE) channel estimation $\hat{h}=\frac{\sqrt{\rho_{\tau}}}{1+\rho_{\tau}} y_{1} \sim \mathcal{C N}\left(0, \frac{\rho_{\tau}}{1+\rho_{\tau}}\right)$. The receiver then applies the QAM demapper on the equalized symbols

$$
\hat{x}_{i}^{\mathrm{zf}}=\frac{1}{\sqrt{\rho T-\rho_{\tau}} \hat{h}} y_{i}, \quad \hat{x}_{i}^{\mathrm{mmse}}=\frac{\sqrt{\rho T-\rho_{\tau}} \hat{h}^{*}}{\left(\rho T-\rho_{\tau}\right)|\hat{h}|^{2}+1} y_{i},
$$

$i=2, \ldots, T$, with zero-forcing $(\mathrm{ZF})$ and MMSE equalizers respectively.

\section{A Non-COHERENT CODEBOOK ON THE GRASSMANNIAN OF LINES}

\section{A. Cube-split codebook}

We have seen that the optimal input achieving the capacity $C(\rho, T)$ of the channel (2) is an isotropically distributed vector such that its distribution is invariant under rotation, i.e.,

$$
p(\boldsymbol{x})=p(\boldsymbol{Q} \boldsymbol{x}),
$$

\footnotetext{
${ }^{1}$ We do not restrict to Rayleigh fading in this paper.
}

for any deterministic $T \times T$ unitary matrix $\boldsymbol{Q}$. That is, $\boldsymbol{x}$ is uniformly distributed on the Grassmannian of lines $G\left(\mathbb{C}^{T}, 1\right)$. By definition, $\boldsymbol{x}$ and $h \boldsymbol{x}$ are identical in $G\left(\mathbb{C}^{T}, 1\right)$. Therefore, Grassmannian signaling guarantees error-free detection without CSI in the absence of the additive noise $\boldsymbol{w}$. When the noise $\boldsymbol{w}$ is involved, since it might not be aligned with $x$, the signal direction is perturbed, and $\boldsymbol{y}$ can be dragged away from $\boldsymbol{x}$ with respect to the chordal distance measure. If the codewords are sufficiently distant, it is likely that $y$ is still within the decision region centered at $x$, facilitating correct detection. Therefore, a good Grassmannian constellation must be a collection of vectors on $G\left(\mathbb{C}^{T}, 1\right)$ with maximum pairwise distance.

For a given constellation size $|\mathcal{C}|$, a commonly used codebook design criteria is to maximize the minimum distance between two distinct codewords, i.e,

$$
\max _{\mathcal{C}} \min _{1 \leq j<k \leq|\mathcal{C}|} d\left(\boldsymbol{x}_{j}, \boldsymbol{x}_{k}\right)
$$

It was shown that constellations with maximum minimum distance have low overall error probability, especially for a small number of transmit antennas [4]. A direct numerical solution of (6) would generate an optimal codebook without any structure. Due to the lack of structure, this kind of codebook is normally used with maximum-likelihood decoding. Furthermore, it needs to be stored at both ends of the channel. These complexity and storage requirements hinder the use of unstructured codebooks in practice. As we go for a practical codebook, we would rather relax (slightly) the minimum distance optimality requirement (6) to have a structured codebook that satisfies the implementation constraints on complexity and storage, while preserving good packing properties.

In this work, we propose to use the quantization codebook recently introduced in [8], so-called cube-split codebook, as a constellation for communication. This codebook is structured, requires no storage, and has high packing efficiency. It can be constructed with $T \times 2^{2 B_{0}(T-1)}$ codewords, where $B_{0}$ is a positive integer. Thus, each codeword can be given a binary label of

$$
N=\left\lceil\log _{2}(T)\right\rceil+2 B_{0}(T-1)
$$

bits. In the rest of the paper, we refer to the cube-split codebook as $C S\left(T, B_{0}\right)$ codebook whenever the parameters $T$ and $B_{0}$ need to be specified. The following proposition gives the minimum distance of $C S\left(T, B_{0}\right)$ codebook.

Proposition 1. The minimum distance of the $C S\left(T, B_{0}\right)$ noncoherent codebook is given by

$$
d_{\min }\left(T, B_{0}\right)=\sqrt{1-\left|\frac{B+A\left(\frac{m_{0}+\mathrm{i} m_{1}}{\left|m_{0}+\mathrm{i} m_{1}\right|}\right)^{2}}{B+A}\right|^{2}}
$$

where $m_{0}:=\mathcal{N}^{-1}\left(2^{-B_{0}-1}\right), m_{1}:=\mathcal{N}^{-1}\left(\frac{1}{2}+2^{-B_{0}-1}\right)$, with $\mathcal{N}(x):=\frac{1}{\sqrt{2 \pi}} \int_{-\infty}^{x} e^{-\frac{t^{2}}{2}} d t$, and

$$
A:=\frac{1-\exp \left(-\frac{m_{0}^{2}+m_{1}^{2}}{2}\right)}{1+\exp \left(-\frac{m_{0}^{2}+m_{1}^{2}}{2}\right)}, \quad B:=1+(T-2) \frac{1-e^{-m_{0}^{2}}}{1+e^{-m_{0}^{2}}} .
$$


We omit the proof of Proposition 1 due to the lack of space.

Lemma 1. The minimum distance $\delta$ of the optimal codebook $C$ of cardinality $|\mathcal{C}|$ on the complex Grassmannian of lines $G\left(\mathbb{C}^{T}, 1\right)$ is bounded by

$$
2|\mathcal{C}|^{-\frac{1}{2(T-1)}} \geq \delta \geq|\mathcal{C}|^{-\frac{1}{2(T-1)}} .
$$

Proof. On $G\left(\mathbb{C}^{T}, 1\right)$ with normalized invariant measure $\mu($.$) ,$ the volume of a metric ball $\mathcal{B}(\delta)$ of radius $\delta$ is given by [9]

$$
\mu(\mathcal{B}(\delta))=\delta^{2(T-1)}
$$

Let $C$ denotes the optimal codebook on $G\left(\mathbb{C}^{T}, 1\right)$ with minimum distance $\delta$, we have the Gilbert-Varshamov lower bound and Hamming upper bound on the size of the code:

$$
\frac{1}{\mu(\mathcal{B}(\delta / 2))} \geq|\mathcal{C}| \geq \frac{1}{\mu(\mathcal{B}(\delta))}
$$

Next, by substituting (10) into (11), (9) follows readily.

In Fig. 1, we plot these fundamental bounds and the minimum distance of $\mathrm{CS}\left(T, B_{0}\right)$ codebook given in Proposition 1 for $T=2$ and $T=4$. We also show the minimum distance of other structured Grassmannian constellations, namely, the Fourier codebook in [6] and the exponential-mapped codebook in [7]. From the figure, it is clear that the cube-split codebook has greater minimum distance than the two other codebooks.

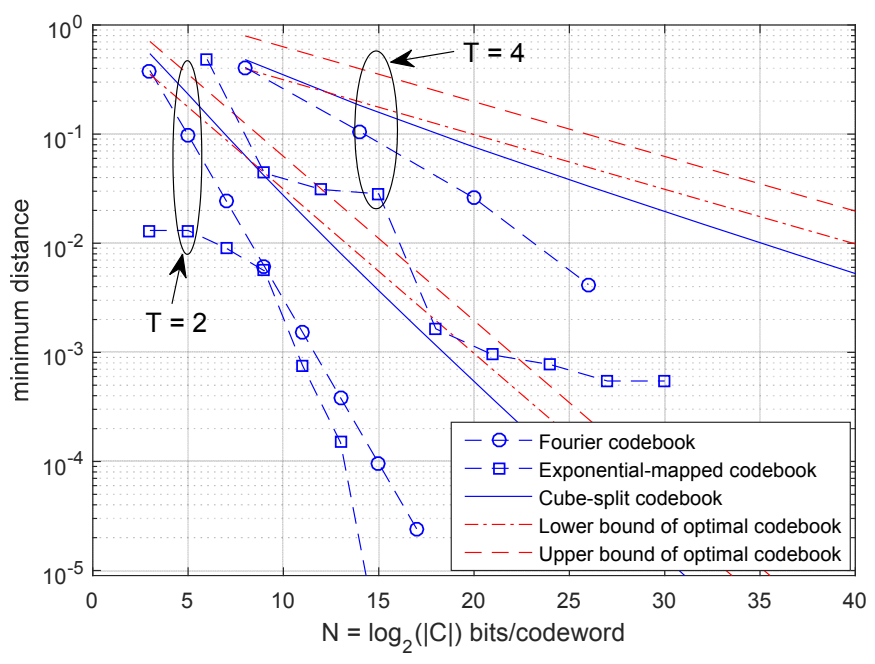

Fig. 1. The minimum distance vs. codebook size of cube-split codebook in comparison with other codebooks and the fundamental limits of optimal codebook. In the case $T=4$, due to the the lack of analytical expression, we only show an upper bound of the minimum distance of the Fourier codebook [6] and the exponential-mapped codebook [7] obtained by a random search.

Next, in Fig. 2, we plot the spectrum of the codeword-wise minimum distance, i.e., the distance from a given codeword to its nearest neighbor, of the $C S(2,4)$ and $C S(4,1)$ codebooks. It can be seen that the codeword-wise minimum distances of these codebooks are localized and compare well to the fundamental bounds. Every codeword in the $C S(4,1)$ codebook even has the same distance to its nearest neighbor, which is $d_{\min }(4,1)$. This property holds for any $C S(T, 1)$ codebooks.

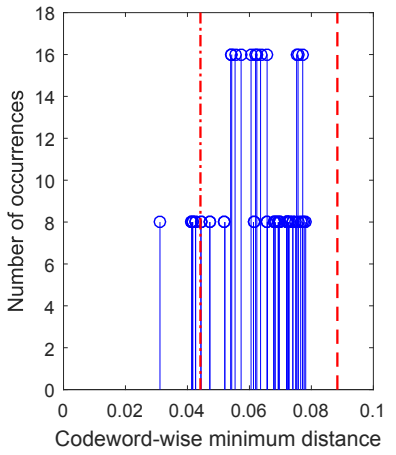

(a) $C S(2,4)$ codebook

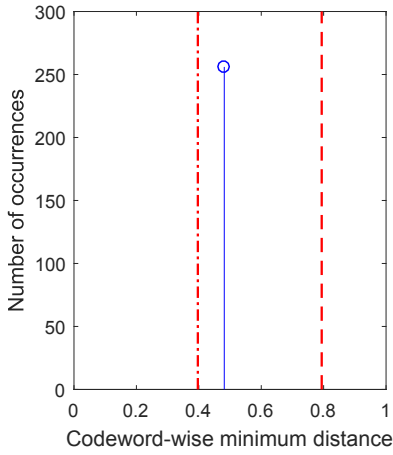

(b) $C S(4,1)$ codebook
Fig. 2. The codeword-wise minimum distance spectrum of $C S(2,4)$ and $C S(4,1)$ codebooks with 512 and 256 codewords, respectively. The dashed and dash-dotted lines are upper bound and lower bound, respectively, of the minimum distance of the optimal codebook of the same size, given in Lemma 1.

\section{B. Maximum likelihood decoder}

The ML decoder is

$$
\hat{\boldsymbol{x}}=\arg \max _{\boldsymbol{x} \in \mathcal{C}} p(\boldsymbol{y} \mid \boldsymbol{x}) .
$$

Given $\boldsymbol{x}, \boldsymbol{y}$ is a Gaussian vector with zero mean and covariance matrix $\mathbb{E}\left[\boldsymbol{y} \boldsymbol{y}^{\mathrm{H}} \mid \boldsymbol{x}\right]=\mathbf{I}_{T}+\rho \boldsymbol{T} \boldsymbol{x} \boldsymbol{x}^{\mathrm{H}}$, hence the likelihood function can be derived as

$$
\begin{aligned}
p(\boldsymbol{y} \mid \boldsymbol{x}) & =\frac{\exp \left(-\boldsymbol{y}^{\mathrm{H}}\left(\mathbf{I}_{T}+\rho T \boldsymbol{x} \boldsymbol{x}^{\mathrm{H}}\right)^{-1} \boldsymbol{y}\right)}{\pi^{T} \operatorname{det}\left(\mathbf{I}_{T}+\rho T \boldsymbol{x} \boldsymbol{x}^{\mathrm{H}}\right)} \\
& =\frac{\exp \left(-|\boldsymbol{y}|^{2}+\frac{\rho T}{1+\rho T}\left|\boldsymbol{y}^{\mathrm{H}} \boldsymbol{x}\right|^{2}\right)}{\pi^{T}(1+\rho T)} .
\end{aligned}
$$

Thus, the ML decoder is simply

$$
\hat{\boldsymbol{x}}=\max _{\boldsymbol{x} \in \mathcal{C}}\left|\boldsymbol{y}^{\mathrm{H}} \boldsymbol{x}\right| .
$$

Following the footsteps of [2], we can derive the pairwise error probability of the ML decoder

$$
P_{p e}^{M L}\left(\boldsymbol{x}_{i} \rightarrow \boldsymbol{x}_{j}\right)=\frac{1}{2}\left[1-\left(1+\frac{4(1+\rho T)}{\left(d\left(\boldsymbol{x}_{i}, \boldsymbol{x}_{j}\right) \rho T\right)^{2}}\right)^{-\frac{1}{2}}\right] .
$$

\section{Log-likelihood Ratio And AChievable Rate}

Let us now consider the use of this codebook together with a channel code. We assign to each codeword of $C S\left(T, B_{0}\right)$ codebook a $N$-bit labeling $\left[b_{1} b_{2} \ldots b_{N}\right]$ in the same manner as in [8]. We assume uniform input probabilities, i.e., all the codewords are equally likely to be transmitted, and so are the bits. The log-likelihood ratio (LLR) of bit $b_{i}$ given the observation $\boldsymbol{y}$ is

$$
\begin{aligned}
\operatorname{LLR}_{i}(\boldsymbol{y}) & =\log \frac{\operatorname{Pr}\left(b_{i}=1 \mid \boldsymbol{y}\right)}{\operatorname{Pr}\left(b_{i}=0 \mid \boldsymbol{y}\right)} \\
& =\log \frac{\sum_{\boldsymbol{\alpha} \in \mathcal{C}_{i}^{(1)}} \operatorname{Pr}(\boldsymbol{y} \mid \boldsymbol{x}=\boldsymbol{\alpha})}{\sum_{\boldsymbol{\beta} \in \mathcal{C}_{i}^{(0)}} \operatorname{Pr}(\boldsymbol{y} \mid \boldsymbol{x}=\boldsymbol{\beta})},
\end{aligned}
$$


where $\mathcal{C}_{i}^{(b)}, b=0,1$, denotes the set of all possible codewords in $C S\left(T, B_{0}\right)$ such that $b_{i}=b$. Applying (14), we have

$$
\begin{aligned}
\operatorname{LLR}_{i}(\boldsymbol{y}) & =\log \frac{\sum_{\boldsymbol{\alpha} \in \mathcal{C}_{i}^{(1)}} \exp \left(\frac{\rho T}{1+\rho T}\left|\boldsymbol{y}^{\mathrm{H}} \boldsymbol{\alpha}\right|^{2}\right)}{\sum_{\boldsymbol{\beta} \in \mathcal{C}_{i}^{(0)}} \exp \left(\frac{\rho T}{1+\rho T}\left|\boldsymbol{y}^{\mathrm{H}} \boldsymbol{\beta}\right|^{2}\right)} \\
& \approx \frac{\rho T}{1+\rho T}\left(\max _{\boldsymbol{\alpha} \in \mathcal{C}_{i}^{(1)}}\left|\boldsymbol{y}^{\mathrm{H}} \boldsymbol{\alpha}\right|^{2}-\max _{\boldsymbol{\beta} \in \mathcal{C}_{i}^{(0)}}\left|\boldsymbol{y}^{\mathrm{H}} \boldsymbol{\beta}\right|^{2}\right) .
\end{aligned}
$$

by using the approximation $\log \left(\sum_{i} \exp \left(x_{i}\right)\right) \approx \max _{i} x_{i}$. We note that the bits in different position have different error protection, and therefore different LLR distributions. This can be seen in Fig. 3, where we depict the LLR histogram of the first 4 bits, given that 0 was sent, for the $C S(2,3)$ codebook.

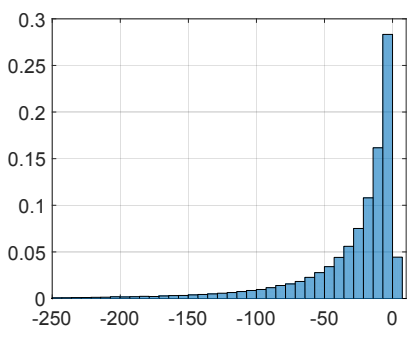

(a) $1^{\text {st }}$ bit

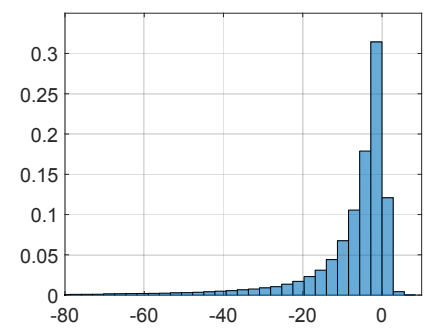

(c) $3^{\text {rd }}$ bit

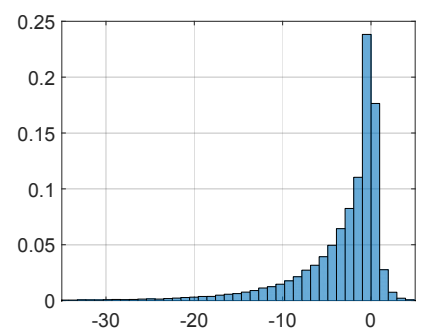

(b) $2^{\text {nd }}$ bit

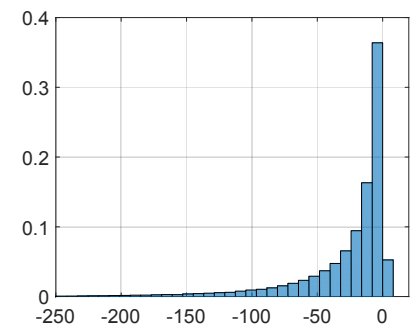

(d) $4^{\text {th }}$ bit
Fig. 3. The histogram of the LLR of the first 4 bits, given that 0 was sent, using $C S(2,3)$ codebook, which has 128 codewords, i.e., 7 bits/codeword. Please note the difference in the horizontal scale of the sub-figures.

Next, we compute the achievable rate achieved with the cube-split codebook. Under the assumption of uniform input probabilities, the average mutual information of bit $b_{i}$ and its $\log$-likelihood ratio $\operatorname{LLR}_{i}(\boldsymbol{y})$ is given by

$$
\begin{aligned}
& I\left(b_{i} ; \operatorname{LLR}_{i}(\boldsymbol{y})\right)=\mathbb{E}_{b_{i}, \operatorname{LLR}_{i}(\boldsymbol{y})}\left[\log _{2} \frac{p\left(\operatorname{LLR}_{i}(\boldsymbol{y}) \mid b_{i}\right)}{p\left(\operatorname{LLR}_{i}(\boldsymbol{y})\right)}\right] \\
& \quad=\frac{1}{2} \sum_{b=0}^{1} \mathbb{E}_{\mathrm{LLR}_{i}(\boldsymbol{y})}\left[\log _{2} \frac{2 p\left(\operatorname{LLR}_{i}(\boldsymbol{y}) \mid b_{i}=b\right)}{\sum_{b_{0}=0}^{1} p\left(\operatorname{LLR}_{i}(\boldsymbol{y}) \mid b_{i}=b_{0}\right)}\right],
\end{aligned}
$$

which can be estimated empirically using the Monte Carlo method. By ignoring the correlation between the LLRs, a lower bound on the achievable rate is then

$$
R=\frac{1}{T} \sum_{i=1}^{N} I\left(b_{i} ; \operatorname{LLR}_{i}(\boldsymbol{y})\right) \quad \text { bits/channel use. }
$$

Similarly, we can calculate the achievable rate of the trainingbased codebook with QAM input in Section II-A. With ZF equalizer $\hat{x}_{i}=\frac{y_{i}}{\sqrt{\rho T-\rho_{\tau}} h}, i=2, \ldots, T$, the likelihood function of the equalized symbol $\hat{x}_{i}$ is

$$
p\left(\hat{x}_{i} \mid x_{i}\right)=\frac{\frac{1+\rho_{\tau}}{\rho_{\tau}\left(\rho T-\rho_{\tau}\right)}+\frac{\left|x_{i}\right|^{2}}{\rho_{\tau}}}{\pi\left(\frac{1+\rho_{\tau}}{\rho_{\tau}\left(\rho T-\rho_{\tau}\right)}+\frac{\left|x_{i}\right|^{2}}{\rho_{\tau}}+\left|\hat{x}_{i}-x_{i}\right|^{2}\right)^{2}} .
$$

Let us denote $x_{\{i\}}$ the transmitted QAM symbol accounting for the bit $b_{i}, i=1, \ldots, N$, and $\hat{x}_{\{i\}}$ the estimation of $x_{\{i\}}$ at the receiver (after equalization), then the LLR of the bit $b_{i}$ is

$$
\begin{aligned}
\operatorname{LLR}_{i}^{\text {training }}\left(\hat{x}_{\{i\}}\right) & =\log \frac{\operatorname{Pr}\left(b_{i}=1 \mid \hat{x}_{\{i\}}\right)}{\operatorname{Pr}\left(b_{i}=0 \mid \hat{x}_{\{i\}}\right)} \\
& =\log \frac{\sum_{\alpha \in \mathcal{Q}_{i}^{(1)}} p\left(\hat{x}_{\{i\}} \mid x_{\{i\}}=\alpha\right)}{\sum_{\beta \in \mathcal{Q}_{i}^{(0)}} p\left(\hat{x}_{\{i\}} \mid x_{\{i\}}=\beta\right)},
\end{aligned}
$$

where $\mathcal{Q}_{i}^{(b)}, b=0,1$, denotes a subset of the chosen QAM constellation such that $b_{i}=b$. The achievable rate of the training-based scheme is then calculated similarly to (22).

In Fig. 4, we compare the achievable rate of cube-split codebook given in (22) with the high-SNR channel capacity given in (1) and the rate achieved with training-based scheme with equal power allocation $\rho_{\tau}=\rho$, QAM input, and ZF equalizer. The cube-split codebook can achieve higher rate than the training-based scheme at a given SNR.

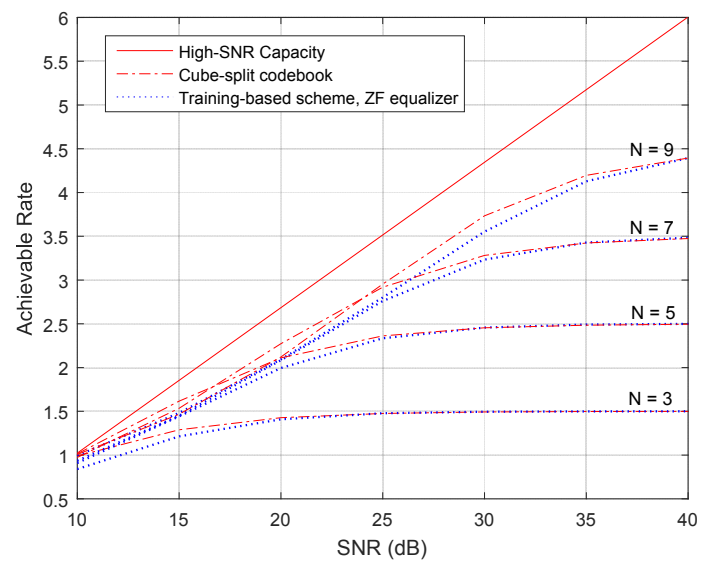

Fig. 4. The achievable rate achieved with cube-split codebook in comparison with channel capacity given in (1), and the achievable rate of the training-based scheme with QAM input for $T=2$ and $N=3,5,7$ and 9 bits/codeword.

\section{ERROR PERFORMANCE}

In this section, we compare numerically the error rates of our cube-split codebook and other structured Grassmannian codebooks, as well as the training-based scheme with equal power allocation $\rho_{\tau}=\rho$. To be fair, in all the figures, we compare different codebooks of the same size, i.e., the same number of bits per codeword. Here, we consider Rayleigh fading $h \sim \mathcal{C N}(0,1)$.

1) Error rate of uncoded codebooks: First, in Fig. 5, we plot the codeword error rate of the cube-split codebook, the Fourier codebook [6], the exponential-mapped codebook [7], and the training-based scheme when $T=2$. While the 
Fourier codebook and the exponential-mapped codebook are worse than the training-based scheme, which spends half of the time resource for channel estimation, our cube-split codebook outperforms the training-based scheme. The cubesplit codebook is also better in terms of bit error rate, as shown in Fig. 6, where the training-based scheme uses Gray labeling for QAM symbols.

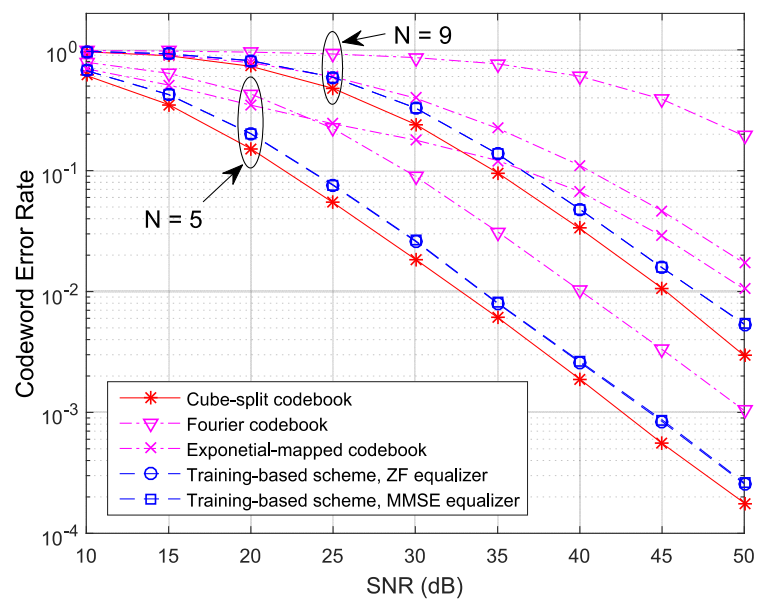

Fig. 5. Codeword error rate of cube-split codebook vs. other structured codebooks and training-based scheme for $T=2$ and $N=5$ and 9 bits/codeword.

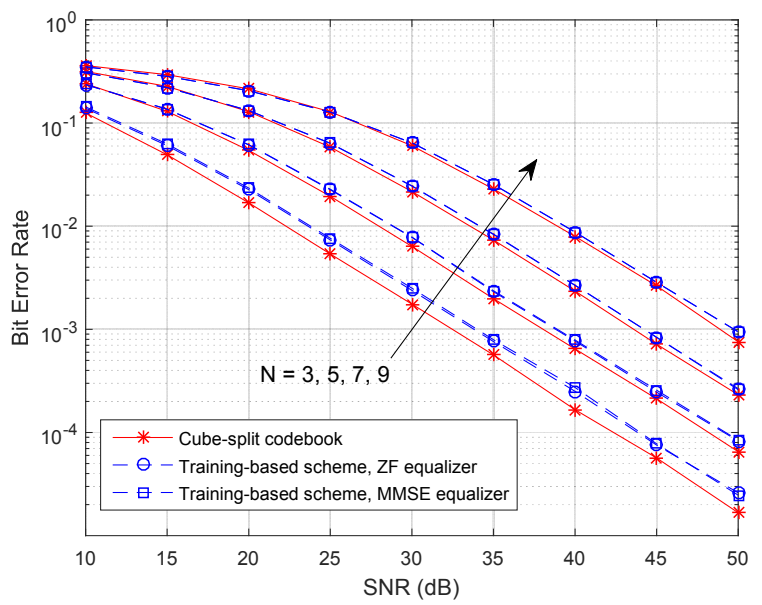

Fig. 6. Bit error rate of cube-split codebook vs. training-based scheme for $T=2$ and $N=3,5,7$, and 9 bits/codeword.

2) Performance with channel coding: Next, we integrate a systematic parallel concatenated rate- $1 / 3$ turbo code with two identical convolutional encoders. The turbo encoder takes in each packet of 640 bits. The turbo decoder calculates the LLR of the received encoded bits and performs 10 iterations of decoding for each bit block.

Fig. 7 presents the bit error rate of the coded cube-split codebook in comparison with coded training based scheme with the same number of uncoded bit per codeword. Having observed that the performance of training-based scheme with ZF equalizer and MMSE equalizer are very close, we consider only ZF equalizer for the simplicity of LLR calculation. As can be seen, in spite of the uneven LLR distribution across the bits, turbo code works well for cube-split codebook and enhances its advantage over the training-based scheme. The power gain for the same bit error rate can be about $2 \mathrm{~dB}$.

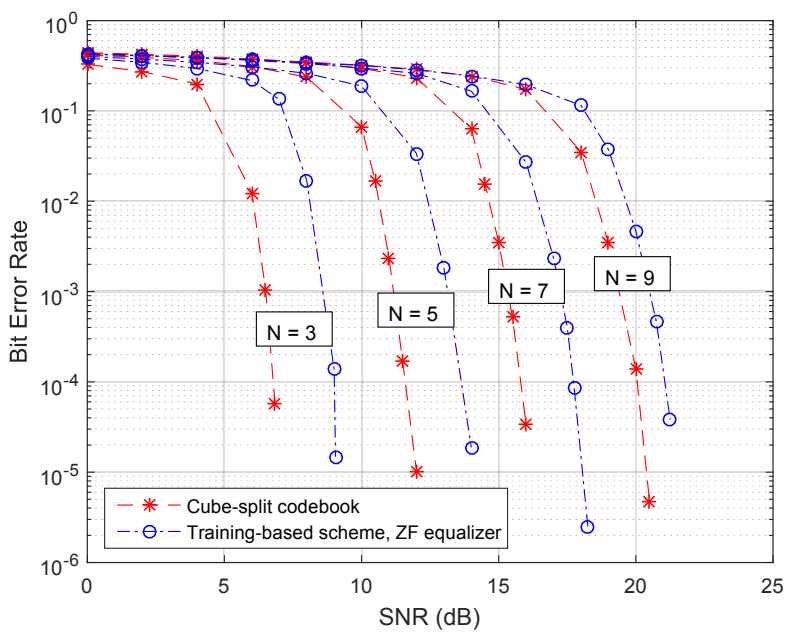

Fig. 7. Bit error rate of cube-split codebook vs. training-based scheme with turbo codes for $T=2$ and $N=3,5,7,9$ bits/codeword.

\section{CONCLUSION}

Aiming for practical application, we have proposed a structured Grassmannian codebook for single-antenna noncoherent communications. Analytical and numerical results show that this codebook has larger minimum distance than other structured codebooks in the literature and outperforms the training-based approach in terms of codeword error rate, bit error rate with/without channel codes, as well as achievable rate.

\section{REFERENCES}

[1] L. Zheng and D. N. C. Tse, "Communication on the Grassmann manifold: A geometric approach to the noncoherent multiple-antenna channel," IEEE Trans. Inf. Theory, vol. 48, no. 2, pp. 359-383, Feb. 2002.

[2] B. M. Hochwald and T. L. Marzetta, "Unitary space-time modulation for multiple-antenna communications in Rayleigh flat fading," IEEE Trans. Inf. Theory, vol. 46, no. 2, pp. 543-564, Mar. 2000.

[3] B. Hassibi and B. M. Hochwald, "How much training is needed in multiple-antenna wireless links?" IEEE Trans. Inf. Theory, vol. 49, no. 4, pp. 951-963, Apr. 2003.

[4] D. Agrawal, T. J. Richardson, and R. L. Urbanke, "Multiple-antenna signal constellations for fading channels," IEEE Trans. Inf. Theory, vol. 47, no. 6 , pp. 2618-2626, Sep. 2001.

[5] R. H. Gohary and T. N. Davidson, "Noncoherent MIMO communication: Grassmannian constellations and efficient detection," IEEE Trans. Inf Theory, vol. 55, no. 3, pp. 1176-1205, Mar. 2009.

[6] B. M. Hochwald, T. L. Marzetta, T. J. Richardson, W. Sweldens, and R. Urbanke, "Systematic design of unitary space-time constellations," IEEE Trans. Inf. Theory, vol. 46, no. 6, pp. 1962-1973, Sep. 2000.

[7] I. Kammoun, A. M. Cipriano, and J. C. Belfiore, "Non-coherent codes over the Grassmannian," IEEE Trans. Wireless Commun., vol. 6, no. 10, pp. 3657-3667, Oct. 2007.

[8] A. Decurninge and M. Guillaud, "Cube-split: Structured quantizers on the Grassmannian of lines," in IEEE Wireless Communications and Networking Conference (WCNC), San Francisco, CA, USA, Mar. 2017.

[9] W. Dai, Y. Liu, and B. Rider, "Quantization bounds on Grassmann manifolds and applications to MIMO communications," IEEE Trans. Inf. Theory, vol. 54, no. 3, pp. 1108-1123, March 2008. 